\title{
Preliminary study on verifying the detection of gait intention based on knee joint anterior displacement of gait slopes
}

\author{
Changho Yu ${ }^{\mathrm{a}}$, Seung Rok Kang ${ }^{\mathrm{a}}$, Giltae Yang ${ }^{\mathrm{a}}$, Chul Un Hong ${ }^{\mathrm{a}}$, Hyung Jong Lee ${ }^{\mathrm{b}}$, Do Young \\ $\mathrm{Oh}^{\mathrm{c}}$ and Tae Kyu Kwon ${ }^{\mathrm{a}}$, \\ ${ }^{a}$ Division of Biomedical Engineering, Chonbuk National University, Jeonju-si, Jeonbuk, Korea \\ ${ }^{b}$ Department of Healthcare Engineering, Chonbuk National University, Jeonju-si, Jeonbuk, Korea \\ ${ }^{c}$ EasyMove Co., Ltd., 230 Anyang-ro, Anyang-si, Gyeonggi-do, Korea
}

\begin{abstract}
This study investigated the feasibility of the Infrared (IR) sensor-based walking aids for detecting the gait intention. To compensate for the defects of Force Sensing Resistors (FSRs) or force sensors, such as the velocity control problem on gait slopes, we used IR sensors to investigate knee joint anterior displacement in order to recognize the gait intention. We also measure leg muscle activities and foot pressure, in order to verify our investigation. We placed two IR sensors on the rollator center to sense left and right leg walking intentions. We took EMG signals of four leg muscles, and analyzed them. Foot pressure analysis parameters were the measured force and mean pressure. We conducted experiments on twenty young healthy adults. The results show that knee joint anterior displacement increases according to gait slope and velocity. We confirm similar results of knee joint anterior displacement through the IR sensors.
\end{abstract}

Keywords: Rollator, foot pressure, EMG, IR sensor

\section{Introduction}

Human aging is characterized by a progressive decline in the ability to perform basic mobility tasks eventually resulting in functional dependence and disability. The muscle power of the elderly tends to significantly deteriorate after the age of 65 , due to aging $[1,2]$. Weakened leg muscles make it difficult for pedestrians to walk properly. Most elderly people with weakened leg muscle use sticks or walking aids in their daily lives [3-6]. In order for the elderly to conveniently and happily lead their daily lives, they should be free from difficulty in walking as the most basic activity. Mobility assisting devices including electronic wheelchairs are the technology needed in the field of welfare instruments as the groups of handicapped and the elderly increase, and the marketability of these devices is increasing. In order to solve issues of convenience and improvement of functions, the necessity for objective and generalized International Organization for Standardization (ISO) regulations, as well as the design required by users, and engineering/technological aspects is increasing [7-9].

\footnotetext{
* Address for correspondence: Tae Kyu Kwon, Department of Biomedical Engineering, Chonbuk National University, Jeonju-si, Jeonbuk, Korea. Tel.: +82-63-270-4066; Fax:+82-63-270-2247; E-mail: kwon10@jbnu.ac.kr.
} 
Intelligent robot type walking aids that are developed to support the lives of the handicapped and the elderly basically support the elderly to walk properly, help them sit down and stand up, and let the elderly move to a destination safely and conveniently with top priority. Representative studies dealing with walking aids for the handicapped and the elderly have been conducted for technology in developing the sensors designed for seamless control of walking aid vehicles of seniors, vehicle movement technology for stably controlling vehicles, and obstacle-avoiding technology for solving the difficulty of the handicapped and the elderly. Such studies tend to help the handicapped and seniors use walking aids more easily [10-16].

The most likely technology of identifying the gait intention by using walking aids is FSR sensors or force sensors $[17,18]$. Such technologies do not cause particular problems for the safety of users when walking on the flat. However, there might be an issue when moving on slopes. In particular, they need to slow down when walking on downward slopes. However, much strength on the hand knob is needed to make walking aids move slowly. Current walking aids might increase the walking speed, causing the risk of accident to increase [19]. In addition, according to one particular study dealing with the movement of lower bodies, two IR sensors attached underneath the walking aids estimated the movement of shins when walking and identified the gait intention. However, there has been a lack of study dealing with gradient conditions [20].

Therefore, this study uses IR sensor-based walking aids to measure the forward displacement of knee joints depending on the gradient conditions when walking, to identify the gait intention. In addition, we verified the results of identifying the gait intention from IR sensors by the result of evaluation of electromyogram and foot pressure.

\section{Experimental methods}

\subsection{Subjects}

Thirty healthy persons were enrolled in the study on a volunteer basis. Twenty volunteers without medical history from injury of exercise within 6 months, and also without experience of exercise within the recent 3 months were randomized in to this experiment (20 males: age $24.3 \pm 1.5$ years; weight $75.6 \pm 4.6 \mathrm{~kg}$; height $174.7 \pm 5.3 \mathrm{~cm}$ ). Prior to the beginning of the examination, conforming to the Declaration of Helsinki (1964), written informed consent was obtained from all subjects.

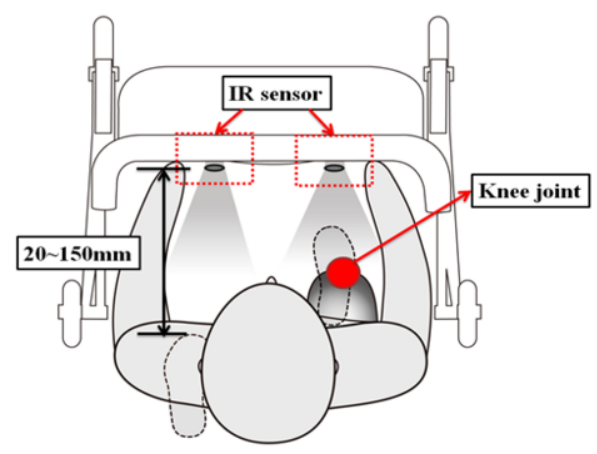

(a)
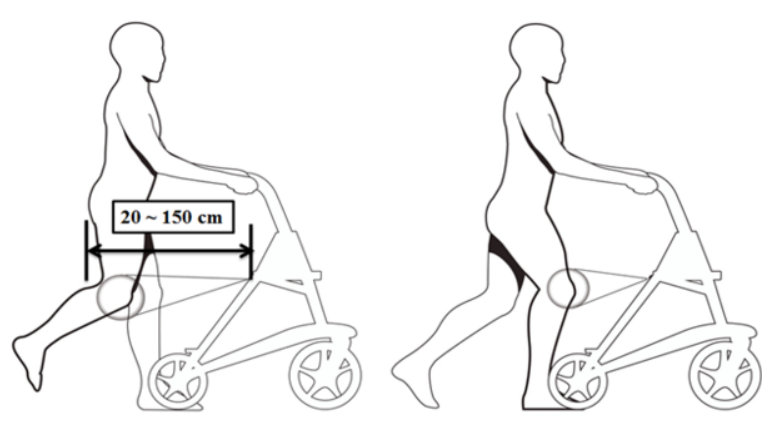

(b)

Fig. 1. Illustrations of knee joint anterior displacement using IR sensor: (a) Top view, (b) Side view. 


\subsection{System configuration}

Figure 1 shows the evaluation of forward displacement of the left and right knee joints by installing two IR sensors (GP2Y0A02YK0F, SHARP Co., Japan) in the middle of the table of an active walker. The active walking aid used in the experiment, Active Walker, is a product that passes the criteria of KPS performed by the Ministry of Commerce, Industry, and Energy, is comprised of ultra-light aluminum materials, and weighs $8.9 \mathrm{~kg}$. Depending on the height of users, the chair height is adjustable from 470 to $620 \mathrm{~mm}$, and the hand knob height is adjustable from 770 to $1000 \mathrm{~mm}$. The IR sensor is an infrared light distance measurement sensor that allows the distance to be adjusted from 20 to $150 \mathrm{~cm}$ for estimation of the knee joints. The operation voltage is analogue output from 4.5 to $5.5 \mathrm{~V}$, and the estimated speed is $38.3 \pm 9.6 \mathrm{~m} / \mathrm{s}$. For defining the forward displacement of knee joint in this study, we measured the value of difference on peak values marked on the IR sensor when moving forward or backwards on the knees when walking to identify the gait intention.

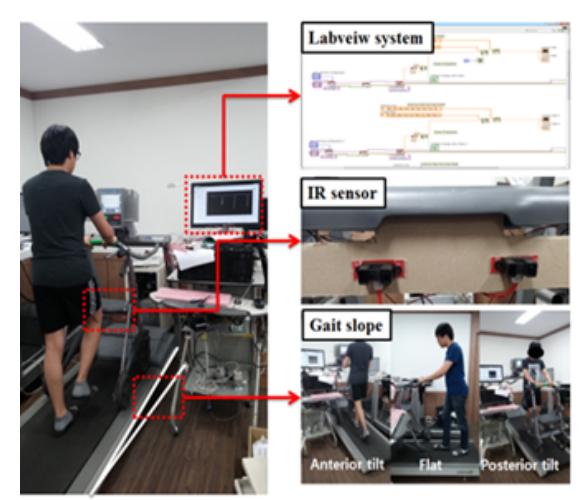

(a)

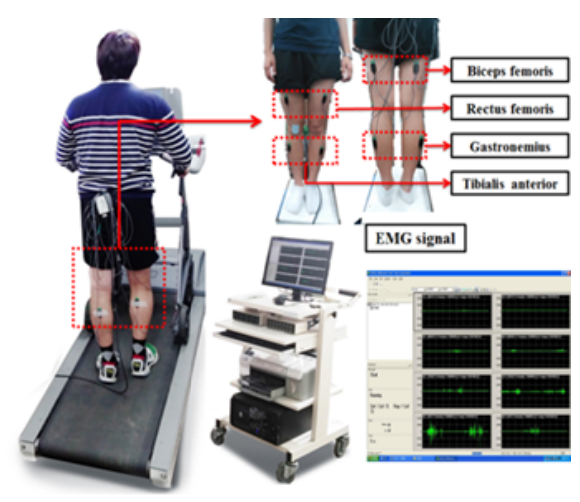

(b)

Fig. 2. (a) Experiment picture for measurement of IR sensors, (b) Experiment picture for measurement of electromyography.

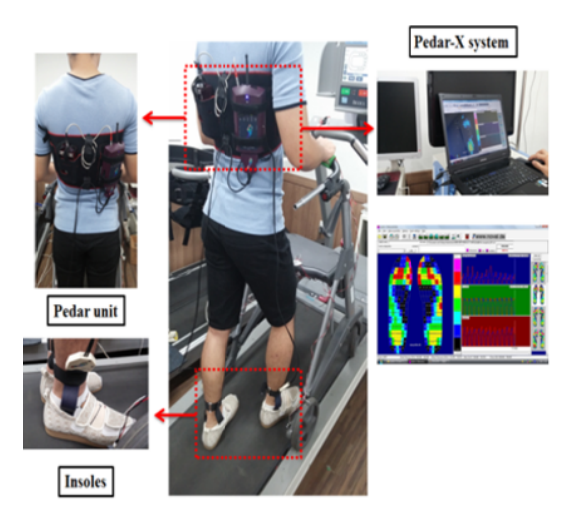

(a)

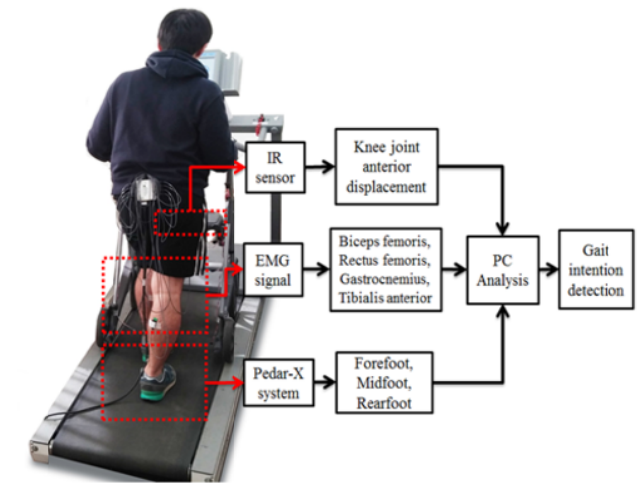

(b)

Fig. 3. (a) Experiment picture for measurement of foot pressure, (b) Algorithm flow for determination of the gait intention. 
For the measurement of forward displacement of the knee joints, we established a baseline for estimation of the IR sensor after attaching markers to both knees. For real-time data transmission, a DAQ board (USB-6009, National Instruments Co., USA) was installed underneath the chair of the walking aid in connection with a computer.

We used NI Labview 2011 (National Instruments Co., USA) system to design the software to identify the gait intention. When the subject starts walking, data of the forward displacement of knee joints of the user were saved, to enable design of a system for identifying walking will through analysis. IR sensor data were estimated for a minute while walking.

Figure 2(a) shows the IR sensor evaluated the forward displacement of the knee joint. Measurement was performed on a treadmill (Gait Trainer, Biodex Ltd., USA) to control the walking speed and gradient conditions. The slope was adjusted (front, back) by using the treadmill. The slope was divided into front slope, rear slope, and flat ground, and the front/rear slopes were 10 degree, while the flat ground was 0 degree. We set the walking speeds to be $1 \mathrm{~km} / \mathrm{h}, 2 \mathrm{~km} / \mathrm{h}$, and $3 \mathrm{~km} / \mathrm{h}$.

We evaluated the muscle activation and foot pressure using IR sensor-based walking aids as shown in Figures 2(b) and 3(a), to compare and analyze physiological signals, and the result derived from the gradient conditions and walking speed when walking by using the IR sensor-based walking aids. The estimated lower limbs muscles were biceps femoris, rectus femoris, gastrocnemius, and tibialis anterior using the Bagnoli-EMG 8ch with the sampling rate of 1000 . In order to remove noise by motion artifact we used a band pass filter from 25 to $450 \mathrm{~Hz}$ and which used sterilization alcohol to reduce the skin resistance against the surface. The standardized EMG signals were conducted for the frequency analysis walking the treadmill the probability density function was earned to calculate the spectral energy to know the muscular activity. Root Mean Square (RMS) analysis was applied to analyze the EMG data for estimating amount of work in muscles during gait.

For foot pressure, we used a Pedar-X system to estimate the fore/mid/hind-feet. We used force and mean pressure as the analysis parameters. Each insole contained 99 force sensors and a working dynamic range of $15-600 \mathrm{kPa}$, which were all calibrated using a standard calibration device. The Pedar-X data acquisition software was used to collect and filter data and sample frequency was set at $100 \mathrm{~Hz}$. Subjects wore the same shoes for the estimation, in order to obtain accurate date value on the foot pressure.

Figure 3(b) indicates the algorithm flow to identify the gait intention. We analyzed the forward displacement of the knee joints value, the muscle activation value, and the foot pressure result obtained from the IR sensors to identify the gait intention, depending on the gradient conditions and walking speed. We performed the experiment for 30 minutes each time for three weeks. In order to remove error due to different postures between subjects, subjects maintained a consistent posture. In addition, all the subjects were well informed of the objectives and method of the experiment prior to it.

\subsection{Statistical analysis}

We intended this study to verify the validity of identifying the gait intention based on the results of IR sensor values for each walking speed and gradient condition, muscle activation, and foot pressure. We analyzed the estimated data for IR sensor value, muscle activation, and foot pressure by using SPSS 18.0 (SPSS Inc., Chicago, IL, USA). We calculated the average and standard deviation of IR sensor values, muscle activation, and foot pressure values for each gradient term and walking speed, 


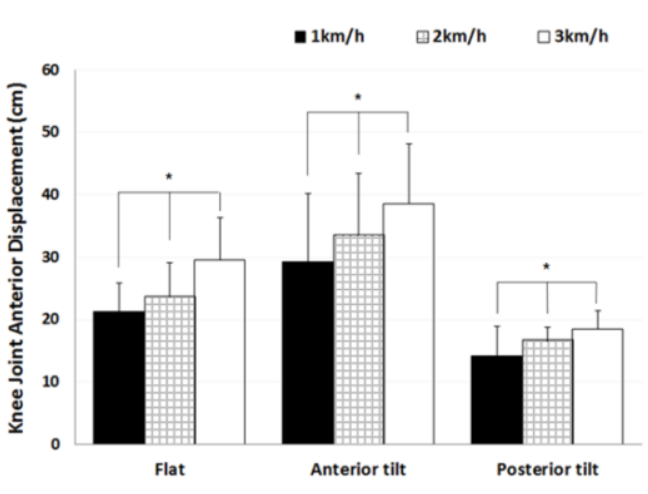

(a)

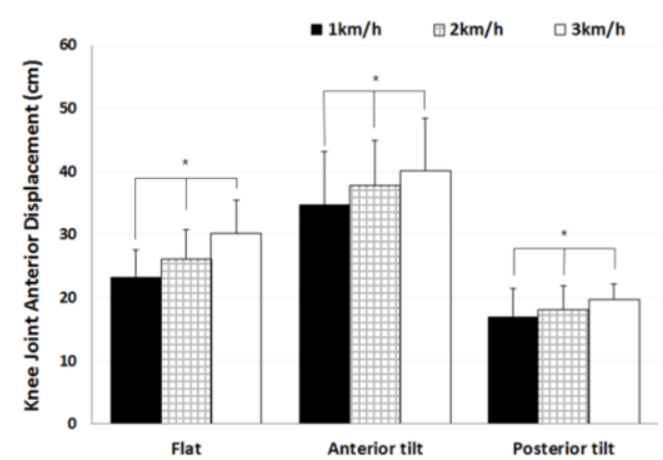

(b)

Fig. 4. (a) Left knee joint anterior displacement for each slope direction and velocity $\left({ }^{*} \mathrm{p}<0.05\right)$, (b) Right knee joint anterior displacement for each slope direction and velocity $\left({ }^{*} \mathrm{p}<0.05\right)$.

and verified all the results with a normality test through the Kolmogorov-Smirnov test. In addition, we performed repeated measure ANOVA to estimate the forward displacement of knee joints, electromyograms, and foot pressure. We set a statistical significant level of $p<0.05$ to verify the significance.

\section{Results}

This study estimated the forward displacement of knee joints according to gradient conditions when walking by using the IR sensor-based walking aids. It was also intended to identify the gait intention. In addition, we verified the results for identifying the gait intention from the IR sensor through the results of estimating the electromyogram and foot pressure.

Using the IR sensor attached to the middle of the table of the walking aid, we estimated the forward displacement of the knee joints, deriving the following results. Figure 4 indicate the results of forward displacement of the left and right knee joints at each walking speed, based on the gradient direction.

According to the result of distance from each of the slopes, speeds, and muscles of lower limb, we found a significant result of the values of forward displacement of the knee joints increasing according to an increase in speed in all of the situations, including level ground, front-inclination, and backinclination. In addition, we found the highest change in knee joints in the case of front-inclination, while obtaining the lowest change in the case of back-inclination. We obtained a similar result in estimation of the muscle biological signals and foot pressure.

Figure 5(a) shows the muscular activity for a $1 \mathrm{~km} / \mathrm{h}$ gait. In the case of the femoral region, the muscular activity increased by about $32 \%$ in the anterior slope, as compared to the posterior slope. In the case of the lower leg, the muscular activity was $8.1 \%$ higher in the posterior slope than in the anterior slope. Figure 5(b) shows the muscular activity for a $2 \mathrm{~km} / \mathrm{h}$ gait. In the case of the femoral region, the muscular activity increased by about $24 \%$ in the anterior slope, compared with the posterior slope. In the case of the lower leg, the muscular activity was $14.7 \%$ higher in the posterior 


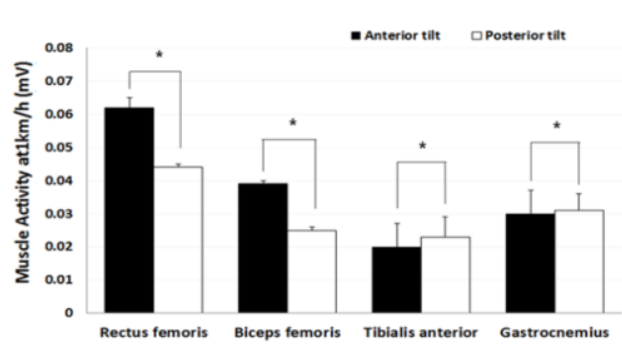

(a)

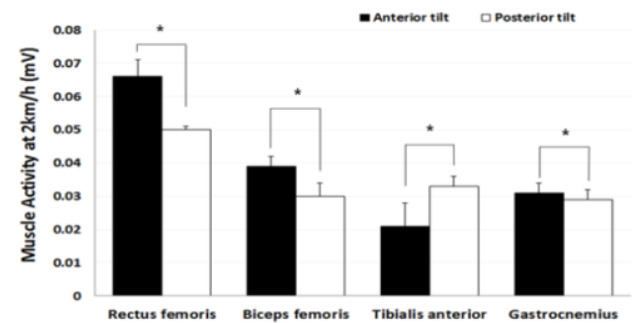

(b)

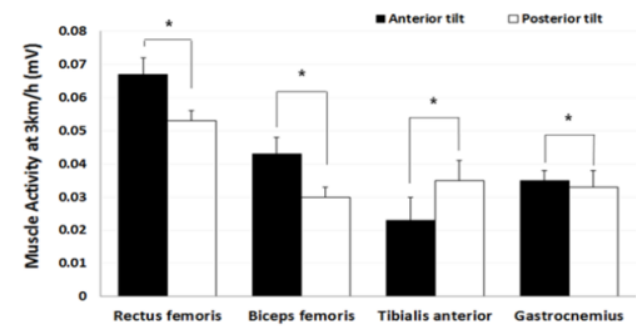

(c)

Fig. 5. (a) Muscular activity with anterior and posterior slopes at $1 \mathrm{~km} / \mathrm{h}\left({ }^{*} \mathrm{p}<0.05\right)$, (b) Muscular activity with anterior and posterior slopes at $2 \mathrm{~km} / \mathrm{h}\left({ }^{*} \mathrm{p}<0.05\right)$, (c) Muscular activity with anterior and posterior slopes at $3 \mathrm{~km} / \mathrm{h}\left({ }^{*} \mathrm{p}<0.05\right)$.

slope than in the anterior slope. Figure 5(c) shows the muscular activity for a $3 \mathrm{~km} / \mathrm{h}$ gait. In the case of the femoral region, the muscular activity increased by about $25.5 \%$ in the anterior slope, as compared with the posterior slope. In the case of the lower leg, the muscular activity was $14.1 \%$ higher in the posterior slope than in the anterior slope.

Figure 6 show the results of force among the real-time foot pressure measurement factors for each situation of gait for anterior and posterior slopes. These show a significant result of the force of the anterior foot varying in accordance with the velocity after supporting the weight of the posterior foot on the anterior slope. For the posterior slope, the force of the posterior foot significantly increased in accordance with the velocity, after supporting the weight of the anterior foot on the posterior slope.

Figure 7 shows the results of mean pressure among the real-time foot pressure measurement factors for each situation of gait for anterior and posterior slopes. These show a similar result to the result associated with the force. We found that the mean pressure of the anterior foot varies in accordance with the velocity, after supporting the weight of the posterior foot on the anterior slope. For the posterior slope, the mean pressure of the posterior foot significantly increased in accordance with the velocity, after supporting the weight of the anterior foot on the posterior slope. 


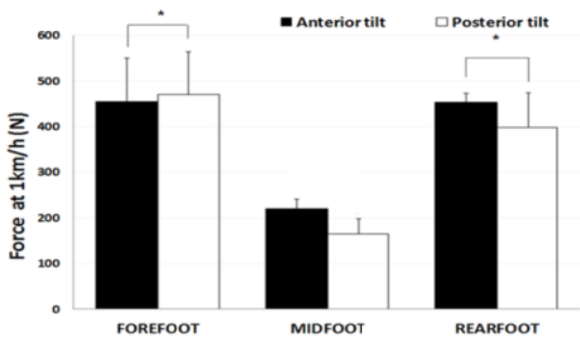

(a)

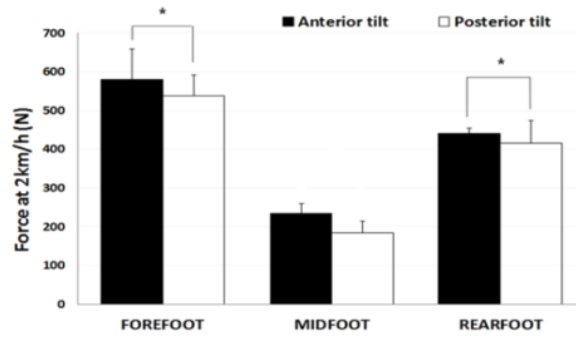

(b)

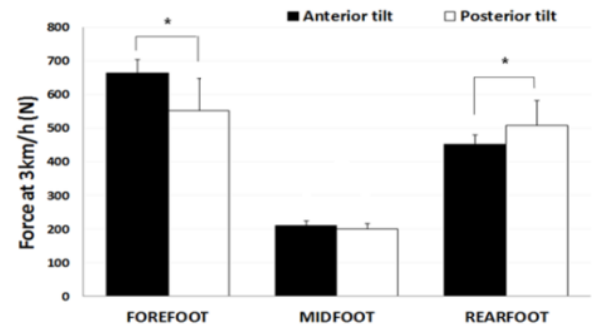

(c)

Fig. 6. (a) Force among the foot pressure factors in accordance with anterior and posterior slopes at $1 \mathrm{~km} / \mathrm{h}\left({ }^{*} \mathrm{p}<0.05\right)$, (b) Force among the foot pressure factors in accordance with anterior and posterior slopes at $2 \mathrm{~km} / \mathrm{h}\left({ }^{*} \mathrm{p}<0.05\right)$, (c) Force among the foot pressure factors in accordance with anterior and posterior slopes at $3 \mathrm{~km} / \mathrm{h}\left({ }^{*} \mathrm{p}<0.05\right)$.

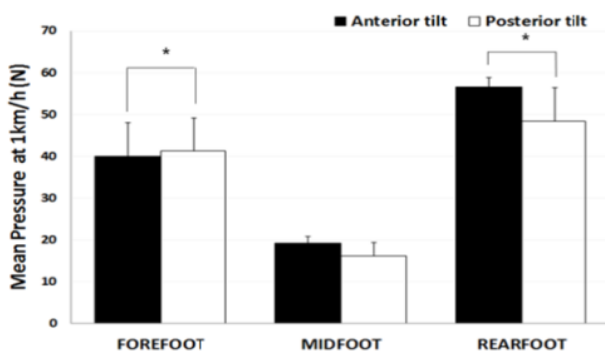

(a)

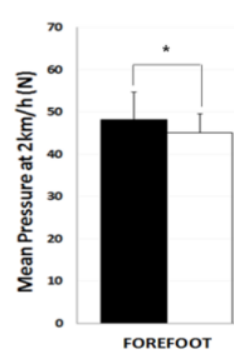

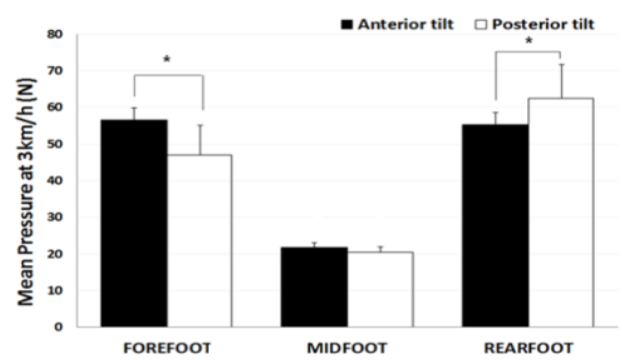

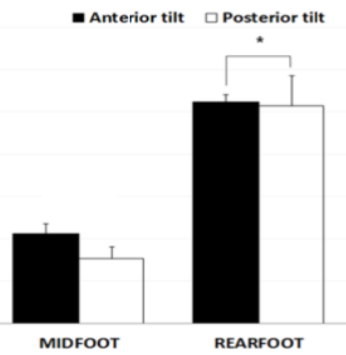

(b)

(c)

Fig. 7. (a) Mean pressure among the foot pressure factors in accordance with anterior and posterior slopes at $1 \mathrm{~km} / \mathrm{h}\left({ }^{*} \mathrm{p}<0.05\right)$, (b) Mean slope among the foot pressure factors in accordance with anterior and posterior slopes at $2 \mathrm{~km} / \mathrm{h}\left({ }^{*} \mathrm{p}<0.05\right),(\mathrm{c})$ Mean pressure among the foot pressure factors in accordance with anterior and posterior slopes at $3 \mathrm{~km} / \mathrm{h}\left({ }^{*} \mathrm{p}<0.05\right)$. 


\section{Discussion}

In this study we found it feasible to identify the gait intention of pedestrians through the simple system of use of the IR sensors. We assumed that identifying the gait intention of pedestrians through the strides of users through IR sensors was more efficient over walking aids that identified the gait intention of users by utilizing a fuzzy algorithm after examining the walking speed and direction of users of previous FSR sensors, or walking aids using handle bars that utilized the adjustable resistance, force sensor, or stretch sensor module $[18,21]$. For walking aids that identify the gait intention in the use of previous FSR sensors, or those utilizing the adjustable resistant handle-bars or force sensors, the speed applied on the walking aids differs, depending on the pressing power. Therefore, there is an issue of overcoming various geographical conditions, except for flatland walking. Furthermore, it is difficult to identify the gait intention, since each of the users has a different amount of strength. However, the forward displacement of knee joints estimates knee joints by using IR sensors while walking. Therefore, it relies on variables shown when walking regardless of pedestrian conditions or requirements, so that it is possible to safely and accurately identify the gait intention of pedestrians [17, $18]$.

For the forward displacement of knee joints, we derived the result that the variable value increased in the knee joints as the speed increased on all the slopes. We assume this to be the result that the stride distance of pedestrians increased from an increase of speed. Such a result turned out to be similar to the result of muscle activation and foot pressure. As the walking speed of front-slope increased, the femoral region muscles were more activated, so that the strength was dispersed on the hind-foot. In the case of the rear-slope, the muscle on the lower legs was more activated according to the speed, in terms of muscle activation. In addition, more of the weight was applied to the forefoot. It appears that the femoral region and hind-foot use more strength to maintain body balance as the center of gravity of the body tilted backwards when walking along the front-slope, and the lower leg and forefoot used more strength to maintain the body balance as the center of gravity of the body tilted forwards when walking down the slope. Pedestrian balance is maintained for safety while walking, as well as to reduce speed when walking on a slope. This seems to be the result of supporting physiological signals to identify the gait intention from the displacement degree of the knee joint muscles when walking on a slope.

\section{Conclusion}

This study used IR sensor-based walking aids to estimate the forward displacement of knee joints according to the slope, and also intended to identify the gait intention. In addition, we verified the result of identifying the gait intention from IR sensors through the result of estimation for electromyogram and foot pressure.

We derived the significant result through the IR sensors that the forward displacement of knee joints depended on the pedestrian conditions and speed when walking on a slope. We showed that the muscle activation of the femoral region increased by $25.5 \%$ when walking on a front-slope, compared to walking on a rear-slope; and the strength was dispersed to the hind-foot. Muscle was activated on the lower leg more than $14.1 \%$ when walking on a rear-slope, than when walking on a front-slope, and the strength was dispersed on the fore-foot.

Moreover, we hope that this result seems to support physiological signals for identifying the gait intention depend on the displacement degree of knee joints when walking on a slope. 


\section{Acknowledgment}

This work was supported by Ministry of Trade, Industry \& Energy (MOTIE) (QoLT Technology Development, No. 10048001).

\section{References}

[1] E. Kennis, S.M. Verschueren, A. Bogaerts, W. Coudyzer, S. Boonen and C. Delecluse, Effects of fitness and vibration training on muscle quality: A 1-year postintervention follow-up in older man, Archives of Physical Medicine and Rehabilitation 94 (2013), 910-918.

[2] C.H Yu, Y.J. Piao, K. Kim and T.K. Kwon, The effects of an 8-weeks training on postural control for the elderly, International Journal of Precision Engineering and Manufacturing 15 (2014), 161-168.

[3] O. Salah, A. Ramadan, S. Sessa and A.A. Abo-Ismail, A systematic approach for design a low-cost mobility assistive device for elderly people, International Journal of Medical, Health, Pharmaceutical and Biomedical Engineering 5 (2011), 36-41.

[4] R. Hari Krishnan and S. Pugazhenthi, Mobility assistive devices and self-transfer robotic systems for elderly a review, Intelligent Service Robotics 7 (2014), 7-49.

[5] C.H. Yu, U.R. Kim and T.K. Kwon, Fundamental study of basal physical fitness and activities of daily living for the aged in relation to indoor horse riding exercise, Bio-Medical Materials and Engineering 24 (2014), 2407-2415.

[6] C.H. Yu, S.R. Kang, H.C. Jeong, K. Kim and T.K. Kwon, Effect of recovery from muscle strength imbalance in lower limb using four point weight bearing reduction system, Bio-Medical Materials and Engineering 24 (2014), 24752483.

[7] R.A. Cooper, B.E. Dicianno, B. Brewer, E. LoPresti, D. Ding, R. Simpson, G. Grindle and H. Wang, A perspective on intelligent devices and environments in medical rehabilitation, Medical Engineering \& Physics 30 (2008), 1387-1398.

[8] C. Schlieder, U. Schmid, M. Munz and K. Stein, Assistive technology to support the mobility of senior citizens, Künstliche Intelligenz 27 (2013), 247-253.

[9] M.M. Martins, C.P. Santos, A. Frizera-Neto and R. Ceres, Assistive mobility devices focusing on smart walkers: Classification and review, Robotics and Autonomous Systems 60 (2012), 548-562.

[10] B. Graf, An adaptive guidance system for robotic walking aids, Journal of Computing and Information Technology 17 (2009), 109-120.

[11] A.C. Lopes, G. Pires and U. Nunes, Assisted navigation for a brain-actuated intelligent wheelchair, Robotics and Autonomous Systems 61 (2013), 245-258.

[12] O. Chuy, Y. Hirata and K. Kosuge, A new control approach for a robotic walking support system in adapting user characteristics, IEEE Transactions on Systems, Man, and Cybernetics, Part C: Applications and Reviews 36 (2006), 725-733.

[13] G. Lacey and K.M. Dawson-Howe, The application of robotics to a mobility aid for the elderly blind, Intelligent Robotics Systems 23 (1998), 245-252.

[14] F.W. Van Hook, D. Demonbreun and B.D. Weiss, Ambulatory devices for chronic gait disorders in the elderly, American Family Physician 67 (2003), 1717-1724.

[15] R. Tan, S. Wang, Y. Jiang, K. Ishida, M.G. Fujie and M. Nagano, Adaptive control method for path-tracking control of an omni-directional walker compensating for center-of-gravity shifts and load changes, International Journal of Innovative Computing, Information and Control 7 (2011), 4423-4434.

[16] Y. Hirata, A. Hara and K. Kosuge, Motion control of passive intelligent walker using servo brakes, IEEE Transactions on Robotics 23 (2007), 981-990.

[17] D.K. Lee, J.S. Kong, M.S. Gohv, S.M. Lee, S.J. Kang and E.H. Lee, Design of the control algorithm for improvement of the convenience the active-type walking aid, Journal of Rehabilitation Welfare Engineering \& Assistive Technology 5 (2011), 17-25.

[18] D.K. Lee, J.W. Lee, M.S. Jang, J.S. Kong and E.H. Lee, A study on walk intention identify method for convenience improvement of walk assistance aids, Journal of Rehabilitation Welfare Engineering \& Assistive Technology 3 (2009), 7-13.

[19] W.Y. Lee, S.H. Eom, M.S. Jang, O.S. Kwon and E.H. Lee, The study of methods for improve the linearity of the walking assistant robot to move on lateral slopes, Journal of the Institute of Electronics Engineers of Korea 50 (2013), 261-268. 
[20] G. Lee, T. Ohnuma and N.Y. Chong, Design and control of JAIST active robotic walker, Intelligent Service Robotics 3 (2010), 125-135.

[21] J.S. Kong, D.K. Lee, Y.S. Nam, B.H. Lee and E.H. Lee, Optimized walking will recognizing system of the walking aid with the fuzzy algorithm, Journal of Korean institute of intelligent systems 18 (2008), 692-699. 